\title{
Basic convective element: bubble or plume? A historical review
}

\author{
J.-I. Yano \\ GAME/CNRS, URA1357, CNRS-INSU-Météo France, Toulouse, France \\ Correspondence to: J.-I. Yano (jiy.gfder@gmail.com) \\ Received: 8 December 2013 - Published in Atmos. Chem. Phys. Discuss.: 5 February 2014 \\ Revised: 2 June 2014 - Accepted: 4 June 2014 - Published: 10 July 2014
}

\begin{abstract}
During the 1950s and 1960s, when intensive investigations on atmospheric moist convection were launched, two possibilities were identified for the basic element of moist convection: bubble and plume. The present paper reviews the investigations of this period, and suggests how the mass-flux convection parameterization formulation emerged from these early investigations. The choice of the steadyplume model as a key ingredient of the mass-flux formulation is especially carefully discussed. Some historical lessons are suggested, especially in the light of the current trend of more emphasis on the bubble dynamics as elucidated by high-resolution numerical modeling and laboratory experiments.
\end{abstract}

\section{Introduction}

The majority of the current operational as well as climateprojection models adopt mass-flux-based convection parameterization, thus a good understanding of its physical basis is imperative for a better understanding of the model behavior. The core of the mass-flux formulation is, geometrically speaking, in placing either an ensemble of plumes or a single plume within a grid box domain. Specifically, Arakawa and Schubert (1974) adopted an entraining-plume model for this purpose. In order to understand the reason for this choice, we need to trace back the investigations on plume dynamics prior to Arakawa and Schubert. The purpose of the present paper is to present such a critical historical review of convection studies before Arakawa and Schubert, in the 1950s and 1960s.

Intensive investigations on atmospheric convection were initiated over the post-war period. This was partially due to the rapid development of aeronautic transports and the associated safety concerns, as well as to military reasons, but also interests in fluid dynamics, considering atmospheric convection as a natural laboratory of convection. From these investigations during a period of the 1940s to 1950 s, the concept of an entraining plume gradually emerged. Extensive laboratory experiments especially were performed in Cambridge in order to mimic atmospheric convection as a part of their efforts to develop an analytical description of atmospheric convection.

In order to understand the style of the investigations of the period, the state of the art of the technology at the time must also be understood. Digital computers were still in their infancy, and they were not widely available, thus people were forced to resort to analytical methods. Simpler analytical theories were always preferred. Combined with an established tradition of fluid mechanics, seeking a similarity solution (or dimensional analysis) was an obvious way to go (Batchelor, 1954).

Under such a circumstance, a natural desire was to identify the basic elements of atmospheric convection (cf., Morton, 1997a), in analogy with eddies in three-dimensional turbulence, or vorticies in two-dimensional flows (cf., Fritsch, 1995), so that advancement could be made by studying the dynamics of these basic elements. Bubble and plume were identified as the two major candidates (cf., Turner, 1969).

The main purpose of the present paper is to review these historical processes of the period with three more specific goals in mind. The first is to provide a systematic literature survey of this subject, because such a self-contained reference list is hard to find in the literature, although reviews of specific subjects exist, as will be pointed out. In the course of the review, basic concepts of the technical terms, some of them already mentioned in the introduction, are also introduced one by one. The second is to examine the implications for the mass-flux convection parameterization developed as an outgrowth of this series of research. Our last goal is, by 
performing a historical review, to try to assess a future direction of research for convection parameterization.

The next two sections review the research from the points of view of bubble and plume, respectively. Observational studies of this period are reviewed in Sect. 4. Consequences and further evolution after the 1960s are outlined in Sect. 5. Issues of stratification are reviewed separately in Sect. 6. The role of the steady-plume hypothesis in mass-flux convection parameterization is examined in Sect. 7, and some future perspectives are remarked on in Sect. 8.

\section{Bubble}

Atmospheric moist convection may be considered consisting of a series of warm bubbles released from the surface level. A cauliflower-like structure seen in clouds may be considered to be a visualization of an ensemble of bubbles. The existence of these warm bubbles (or thermals as they were called) was known about by glider pilots for years. By riding over such a thermal, they were able to boost their gliders substantially.

A good laboratory analog could be a series of air bubbles released from the bottom of a water tank. Such an experiment was originally performed by Davies and Taylor (1950) from fluid-mechanical interests. Their experiment, in turn, induced the interest of an Imperial College group in studying atmospheric moist convection as an ensemble of bubbles.

A major difference from the atmospheric bubbles to air bubbles used in the experiments by Davies and Taylor (1950) is that the former mix gradually with the environment as they ascend, whereas the latter are immiscible. In order to introduce such a mixing tendency, the salt water was taken, instead of air, as a source of bubbles within a water tank (Scorer and Ronne, 1956; Scorer, 1957; Woodward, 1959).

In their experiments, a hemispheric copper cup was filled with dense salt water, which was turned over quickly by hand into a water tank in order to generate a bubble, but in an upside-down manner. The focus of the study was the time evolution of a single bubble, thus only a single bubble was released at one time in all the experiments reported.

An example of such an experiment is shown in Fig. 1: the highly transient nature of the bubble dynamics may be noted. Detailed measurements of the velocity around a bubble (Woodward, 1959) revealed that a doughnut-shaped vortex ring was formed inside the bubble (Fig. 2).

Saunders (1962) examined the behavior of the bubble intruding into a stable "inversion" layer above. Turner (1963a) tried to mimic the latent heat effect by a chemical reaction between hydrochloric acid $(\mathrm{HCl})$ inside a bubble and sodium bicarbonate $\left(\mathrm{NaHCO}_{3}\right)$ in the environment. The reaction generated carbon dioxide that boosted the bubble by associated buoyancy. Under this set-up, the light bubbles are injected from the bottom.

Ludlam and Scorer (1953) reviewed atmospheric convection as a whole from the point of view of bubbles as its ba-

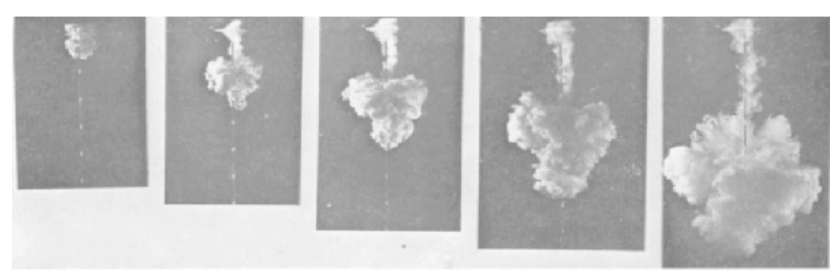

Figure 1. Example of the bubble experiment: photos of a sequence from left to right (reproduced from Scorer (1957)).

sic elements. Scorer and Ludlam (1953) tried to establish the bubble concept for atmospheric convection by examining cloud photo sequences as well as a basic theoretical analysis. Malkus and Scorer (1955) attempted the same for the observed clouds more systematically. Simpson (1983b) provides a personal historical retrospective on her involvement, which also led to her decision to choose a plume model after considerations of both possibilities. In this respect, it appears that Malkus and Scorer (1955) were rather looking for starting plumes, as later conceptualized by Turner (1962), than the isolated bubbles (see Sect. 3 below).

For the theoretical side, Turner (1957) examined the interplay of the vortex-ring dynamics and the role of buoyancy. Turner (1963b) extended the analysis to the case with a bubble (thermal) surrounded by turbulent flows. Here, the magnitude of the surrounding turbulence is measured by a velocity scale.

Levine (1959) was one of the first to consider an idealized bubble model for atmospheric convection in a self-contained manner. More specifically, he considered the vertical motion of an isolated bubble in an infinite domain under a quiescent state at infinity. Under this condition, the most remarkable conclusion is that the dynamic pressure trivially vanishes at the center of the bubble, thus no effect of the dynamic pressure is found in the total vertical momentum equation.

More precisely, Levine (1959) considered a spherical bubble. Hill's vortex solution (Lamb, 1932) is adopted inside the bubble in order to describe a vortex ring structure. The flow outside the spherical bubble is constructed by an irrotational flow assuming a continuity of the tangential velocity at the surface. This inviscid-flow solution is exploited explicitly by Levine in order to derive a drag force acting on the bubble. In order to obtain a drag force, it is assumed that the bottom part of the bubble is open to outside air, where a drag force is inserted.

Turner (1964) expanded Levine's work to the case when the bubble increased in size with time, and examined in more detail the structure of flows inside the bubble.

In spite of its strongly intuitive nature, and also intensive research during the 1950s and 1960s, somehow the bubble theory was eventually taken over by the plume theory. A reason for this shift is hard to say simply from the existing literature. Clearly no final verdict was issued in any published references. It is most likely that the investigations simply shifted 


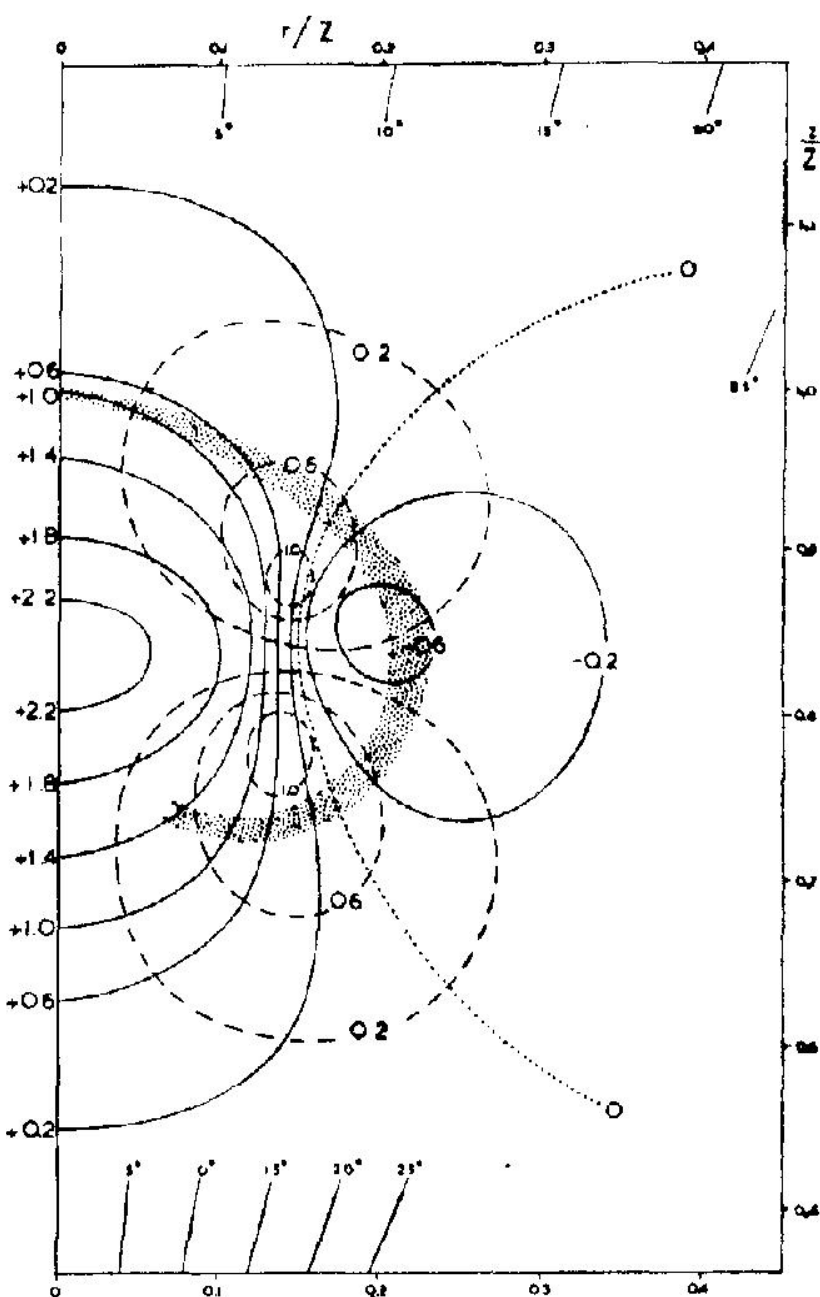

Figure 2. The distribution of velocity in and around an isolated thermal. Only the right-hand side of the thermal is shown. The outline of the buoyant fluid is shaded. The solid and dashed lines show the vertical and radial velocities, respectively, with the values indicated as multiples of the vertical velocity of the thermal cap (reproduced from Fig. 2 of Woodward, 1959).

away due to a difficulty in casting a bubble theory into a selfconsistent steady theory.

Here, as a historically unfortunate legacy of the bubble theory, the formulation by Levine (1959) for the vertical momentum equation was uncritically taken into plume models, originally by Simpson et al. (1965), and this tradition still continues (e.g., Donner, 1993; Bechtold et al., 2001; Siebesma et al., 2003; Bretherton et al., 2004; Zhang et al., 2005). In the plume dynamics, the dynamic pressure is certainly not negligible. Its importance in the convective-plume dynamics is demonstrated by Holton (1973); Soong (1974); Yau (1979); Kuo and Raymond (1980). Fig. 19 of Soong (1974) especially showed that the dynamic pressure force is substantially balanced out by the buoyancy force. However, the term is systematically neglected in the literature by quot- ing Levine (1959), but with a rather ad hoc adjustment such as the introduction of "effective mass".

The drag force derived under elaborate effort by Levine (1959) also becomes irrelevant in applying his formulation to the plume dynamics. In the latter case, it can easily be shown that the drag coefficient will simply be equal to the fractional entrainment rate of a given plume (cf., the definition associated with Eq. 3 below).

\section{Plume: entraining plume model}

The idea of a plume can most vividly be seen by a water-tank experiment originally performed by Morton et al. (1956). They placed a constant buoyant-mass source at the bottom of a water tank (with dyed alcohol as a marker), and examined the resulting motion. The result was a plume gradually growing upwards, which may be considered steady after a substantial time (Fig. 3).

In general, the plume refers to convective flows resulting from a continuous source of buoyancy. They tend to be quasi steady, in contrast to the inherently transient nature of bubbles.

In the case of the original experiment by Morton et al. (1956), the plume grows upwards by sucking the surrounding water at a constant rate, and as a result it also increases its radius at a constant rate with height. Such a "sucking" process is commonly referred to as entrainment. A particular plume solution obtained by them is called the entraining plume, because it is characterized by a constant fractional entrainment rate (cf., Eq. 3 below).

Importantly, the obtained laboratory result is consistent with a theoretical result predicted by Batchelor (1954) using a similarity theory. Here, a similarity theory seeks a form of a solution of a given system that is determined solely by examining the dimensionality of the relevant variables and parameters.

Morton (1957) took a next theoretical step by introducing humidity into the plume model of Morton et al. (1956). This paper is particularly illuminating, because it already suggests a potential limit of taking a plume model for moist convection. He found, by performing an analysis under a relatively limited setting with a constant potential-temperature profile and no density stratification, the tendency of a plume to grow to infinity both in size and height when a bottom plume size is above a critical value: see his Fig. 6 and compare it with the case below this critical value shown in his Fig. 5. Morton (1957) concluded that "When a cloud grows past the critical size it may be taken that the increased buoyancy forces in the centre and the decreased forces at the edge dominate the effects due to the plume. The cloud is then no longer properly a part of the plume, and some asymmetry in its structure or some wind effect may cause it to drift clear, or it may persist when the plume dies down. From the need for continuity, it is apparent that the central upward motions must now be 


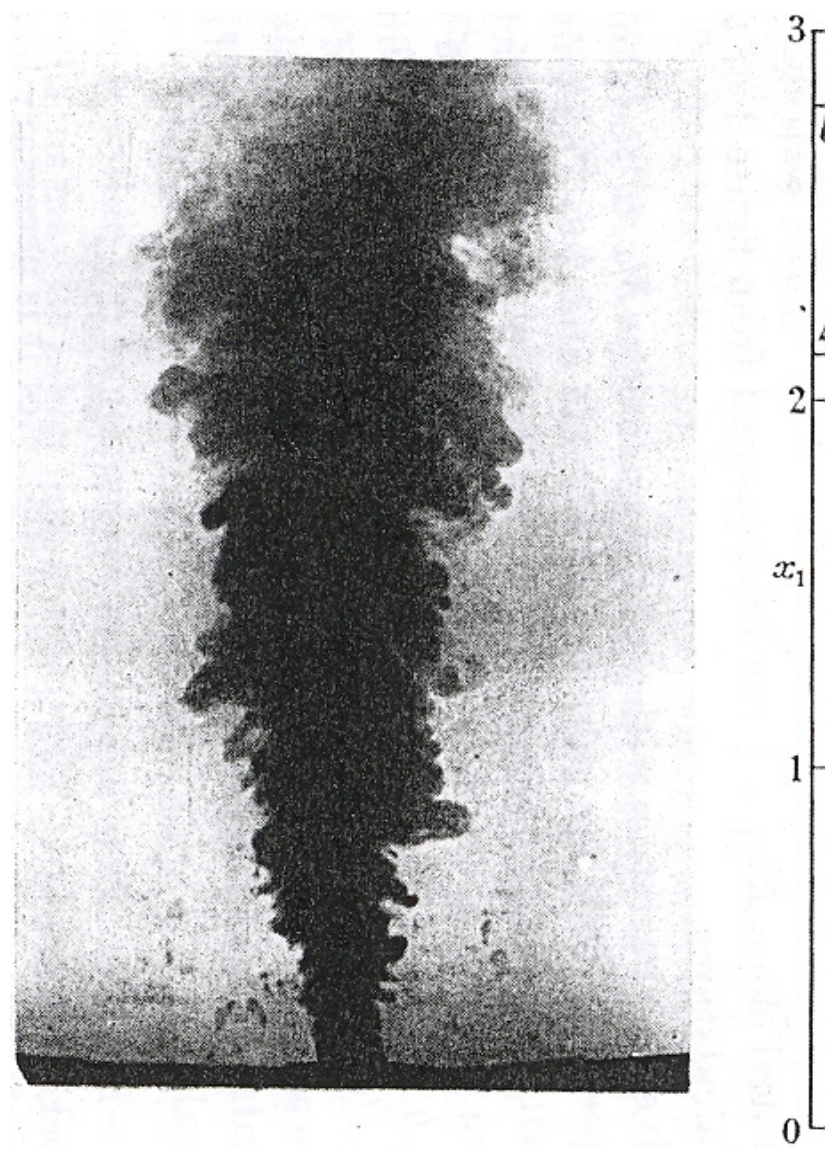

Figure 3. The entraining plume generated by an original water-tank experiment by Morton et al. (1956) (reproduced from Morton et al., 1956).

balanced by downdrafts at the sides, and though the motion in the cloud is turbulent it will possess to some degree the elements of a spherical vortex (not all of which will be marked by condensed water droplets)." Unfortunately, no careful followup study exists to investigate how exactly this explosive tendency of plume under water condensation can be tamed under the presence of stratifications. The issue of stratification is further discussed separately in Sect. 6.

A final important addition was to consider a transient developing phase of a convective plume before it reaches an equilibrium steady state. Such a state was tagged by Turner (1962) as a "starting plume". Under such a transient phase, a top of the plume could still be identified at a finite height. The basic idea here was to add a cap of bubble at the top of an evolving, entraining plume. However, only an outline for this formulation was presented, and the idea was never fully developed in the literature. In the subsequent applications, a starting plume was often interpreted as a prototype for cumulus convection, but under a steady state with a finite height. The Morton et al. (1956) steady plume theory was simply taken as an approximation of it, except for at the cloud-convection top, where it is assumed that all the convec- tive air suddenly exits (i.e., detrains) in a horizontal direction. In general, draining of convective air into a surrounding environment is referred to as detrainment.

The influence of Batchelor's (1954) original work has been considerable. In spite of the fact that the original entraining plume model has been much criticized, as will be seen in Sect. 5 below, the notions of entrainment and detrainment are hardly given up in current convection parameterizations (cf., de Rooy et al., 2013).

\section{Observations}

Note that plume theories were developed assuming that a plume is in steady state. Good agreements with the laboratory experiments were reported in all the literature cited above. Many of them also emphasized applicability to atmospheric convection.

In this historical development, Stommel's (1947, 1951) work on entrainment in trade cumuli stands out as a singular achievement. By mistake or negligence, his papers were not cited by Morton et al. (1956). Stommel's point was that we simply have to assume a lateral mixing with the surrounding air as cloud air ascends, because the buoyancy computed by a moist adiabatic ascent assuming no mixing with the surrounding air far exceeds the observed values. Here he invoked the notion of "entrainment". However, Stommel did not examine whether the convection observed by him was maintained by a continuing buoyancy source as a condition for constituting a "plume", thus a link between his "entrainment" and entrainment observed in a water tank is not obvious. Here we also emphasize that by "entrainment" Stommel merely referred to a lateral mixing of the convective cloudy air with an immediate surrounding, even without specifying how to define this "immediate surrounding".

Then the weather modification experiments came onto the scene in the 1960s. For a review of observational studies prior to these experiments, see Malkus (1952). The decade of the 1960s was a pinnacle of human trust in technology. Everything was believed to be achievable by technology, which culminated in the landing of a human on the moon. It was believed that the weather would be under perfect control in the next century, and serious investments were made towards this direction. A very interesting historical paper to read through, in this respect, is Ludlam (1958).

The basic idea of weather modification was relatively simple: we just sprinkle a small but critical number of particles, which function as ice condensation nuclei such as silver iodide or dry ice, into convective clouds; a catalytic effect would then be induced in the precipitation process, and the cloud would die. Extensive field experiments were performed in order to test this possibility.

In order to verify the experimental results, they must be compared against a result from a numerical model. For this purpose, the entraining plume model was adopted 


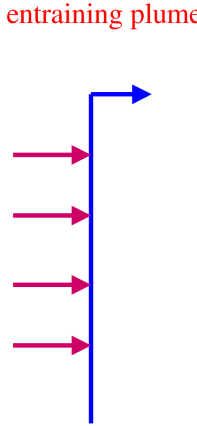

Stommel (1951) cloud top entrainment

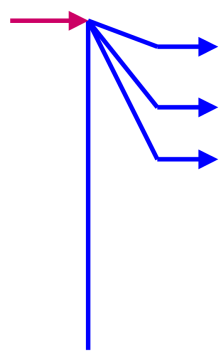

Paluch

(1979) stochastic mixing

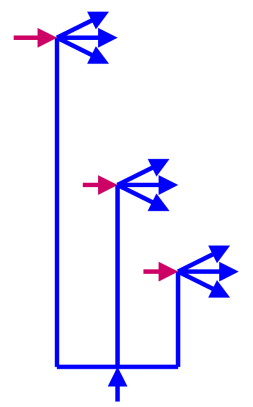

Raymond \& Blyth (1986)
Figure 4. A schematic summary of the three different theories for the atmospheric convective entrainment-detrainment processes. From left to right: Stommel's entraining plume, Paluch's cloudtop entrainment, Raymond and Blyth's stochastic mixing (based on Raymond, 1993).

(Simpson et al., 1965; Simpson and Wiggert, 1969). A preliminary study towards this goal was already made by Malkus (1954). A good agreement between observed, unseeded clouds and numerical predictions was considered to be evidence that the entrainment plume was a good model for convective clouds. Thus we arrive at the point of the work by Arakawa and Schubert (1974).

\section{Historical retrospect: after Arakawa and Schubert}

Such was the history up to the point of Arakawa and Schubert (1974). Arguably, at the moment that Arakawa and Schubert presented their mass-flux formulation, the entrainment plume was probably the most natural choice, though it was far from a unique choice. Here, history is never simply linear. The entraining plume model had already been much criticized on various occasions even before Arakawa and Schubert (1974).

Telford (1966, see also Telford, 1968) pointed out the limits of similarity concepts, especially considering the fact that the experimental plumes are not perfectly steady but associated with extensive transient turbulence, and suggested the importance of "turbulent mixing". This work rectified earlier proposals (e.g., Houghton and Cramer, 1951) for introducing "turbulent" entrainment in addition to standard dynamical entrainment. Here, Telford was not clear on the point of whether he was actually criticizing the original Morton et al. (1956) similarity theory or its application to atmospheric convection.

Morton (1968), in turn, replied to this criticism by focusing on the original similarity theory itself rather than its atmospheric applications. By emphasizing the fact that the theory was based on a time-averaged picture of a plume rather than a transient one, he defended the consistency of his similarity theory. This "time-average" concept is likely to be more important in interpreting atmospheric convection as a steady plume, because the former is highly transient (Stewart Turner, personal communication, 2009). In the case of atmospheric convection, such a transient process is also likely to contribute significantly to the bulk entrainment rate.

Warner (1970) raised extensive criticisms of the entraining plume model. The main criticism was that the model cannot correctly predict both the cloud top (thermal profile of the plume) and liquid water contents at the same time by adjusting the entrainment rate (Warner, 1972). Although the work is often considered a final blow to the entraining-plume hypothesis for atmospheric convection, readers should carefully read the response by an original author (Simpson, 1971, 1972) before convincing themselves.

A major counter-mechanism was proposed by Squires (1958a, b) and Paluch (1979; see also Blyth et al., 1988). They argued based on their "mixing line" analysis that a dominant mixing process is due to penetrative downdrafts from the cloud top (a type of "cloud-top entrainment"). Here, a straight line called a "mixing line" was identified for a scatter plot of in-cloud values of the wet equivalent potential temperature and the total water, the two quantities that are conserved under non-precipitating processes. The simplest interpretation of this "mixing line" was that the air at the cloud bottom and the cloud top mixed together directly.

Telford (1975) further argued that a single "cloud" element may detrain at multiple levels. The last idea led to the proposal of the "stochastic mixing" model by Raymond and Blyth (1986). They argued that, especially for nonprecipitating clouds, aggregates of many parcels move towards buoyancy equilibrium from the cloud bottom. These parcels follow different eventual fates by mixing with the environment at different rates. This model was extended further by Taylor and Baker (1991). Emanuel (1991) developed a full convection parameterization based on stochastic mixing.

A similar, but somehow simpler mixing formulation called "buoyancy sorting" was proposed by Kain and Fritsch (1990). This scheme performs similar multiple mixing with the environment at every vertical level as for stochastic mixing. However, the parcel does not multiply under this formulation. This parameterization was further elaborated on as by Bechtold et al. (2001). The role of this mechanism is under investigation by various groups using large-eddy simulations (cf., de Rooy et al., 2013).

On the whole, the present convective parameterizations have gradually drifted away from the entraining plume model by giving equal importance to detrainment. See for example Derbyshire et al. (2011), which is considered one of the most recent efforts towards the latter goal. Nevertheless, it may seem surprising to find that the basic notions of entrainment and detrainment have hardly been given up even after half a century.

From a historical perspective, the jet was another type of flow that was studied extensively in order to understand the 
entrainment processes. Here the jet refers to a flow that is generated by a continuous source of momentum, in contrast to the plume, which is generated by a buoyancy source.

The physical bases for the entrainment concept applied to jets and the laboratory-experiment plumes are systematically discussed by Turner (1986; see also Townsend, 1970; List, 1982; Reuter, 1986). Turner suggests that the entrainment concept would also be relevant to some of the outdoor processes such as volcano eruptions, atomic bomb explosions, and possibly bush fires. However, the direct relevance of this concept to atmospheric convection is questionable. In this respect, Malkus and Scorer (1955) foresaw that the bubble dynamics would be the "mechanism of entrainment" in cumulus convection.

Morton (1997b) presents the following historical retrospect: "Early works looked at lateral entrainment and took jets and plumes as models for atmospheric convection. Each requires a maintained source, each rises without limit in a homogeneous environment, but has bounded raise in stable environments where the rising stream overshoots and falls back before spreading laterally, and each has a similarity structure determined by source strength alone, at least up to the level where the mean buoyancy falls to zero. The jet is an unrealistic model in an atmosphere where convection is normally buoyant and compact sources of momentum are uncommon; the plume is not a great deal more realistic as its motion and similarity structure up to the level of zero mean buoyancy are determined primarily by the strength of its maintained source, possibly involving both buoyancy and momentum fluxes. The plume appears even less appropriate as latent heat of condensation and the environment is normally stable. A further deficiency of the plume model is that lateral entrainment with its associated turbulent mixing should produce Gaussian profiles. There is, however, some evidence from aeroplane traverses that cloud properties such as liquid water content, droplet populations and droplet spectra are relatively uniform over considerable parts of cloud sections, but change with height, and only narrow zones of gradient are observed near cloud edges. Such profiles are inconsistent with lateral diffusion."

For many, the first half of the above statement may sound trivial, and it may be argued that these limitations of the original plume models are now well known. However, the fact still remains that even today, we somehow stay with this original framework of the steady-plume model by modifying details. We have to consider carefully how long we can keep going under such a "revisionist" stance. The latter half is, I am afraid, not well appreciated, and this is related to the fact that a distinction between stirring and diffusion (cf., Ottino, 1989 ) is not well recognized in the context of atmospheric convection.

\section{Buoyancy parameter: issues of stratification}

As discussed in Sect. 3, the entraining plume model arises naturally under a dimensional analysis for a fluid without stratification. However, once a stratification is introduced to the system, the similarity theory based on dimensional analysis becomes no longer available. For this reason, it is important to consider the role of stratification in the context of the plume dynamics specifically.

An important non-dimensional parameter playing a key role is the buoyancy parameter introduced by Baines (2001):

$B=\frac{Q N^{3}}{g^{\prime 2}}$,

where $Q$ is the initial volume flux per unit area, $\left(\mathrm{m}^{2} \mathrm{~s}^{-1}\right)$, $N$ the buoyancy frequency (Brunt-Vaisala frequency), $\left(\mathrm{s}^{-1}\right)$, and $g^{\prime}$ the initial buoyancy (reduced gravity), $\left(\mathrm{m} \mathrm{s}^{-2}\right)$. Note that $Q$ is a measure of the initial vertical velocity.

In typical plume experiments in the laboratory (cf, Baines, 2002), this parameter $B$ is more than often set to the order of unity by introducing a finite vertical velocity initially. This point must be emphasized: though the literature argues that the initial momentum source is small, it is indeed finite.

This situation is in a great deal of contrast to typical atmospheric situations, in which the initial velocity of a plume is expected to be virtually nonexistent. Here a major ambiguity in developing this argument stems from an ambiguity in defining the "initial velocity" of an atmospheric plume. If we consider it to be an initial velocity provided by non-convective processes in a boundary layer, a typical value for the buoyancy parameter may be estimated as $B \sim 10^{-3}$ as a possible maximum. Here we use the parameters $g^{\prime} \sim g \theta^{\prime} / \theta \sim 10^{-1} \mathrm{~m} \mathrm{~s}^{-2}, N \sim 10^{-2} \mathrm{~s}^{-1}$, and $Q \sim d w \sim 10 \mathrm{~m}^{2} \mathrm{~s}^{-1}$, assuming $g \sim 10 \mathrm{~m} \mathrm{~s}^{-2}, \theta^{\prime} \sim 1 \mathrm{~K}, \theta \sim$ $10^{2} \mathrm{~K}$, an initial vertical scale $d \sim 10^{2} \mathrm{~m}$, and an initial vertical velocity $w \sim 10^{-1} \mathrm{~m} \mathrm{~s}^{-1}$. Though the choice of the above parameters is somewhat arbitrary, the readers can check by themselves how the parameter, $B$, would change by changing these choices. Alternatively, we may take values more typical of a cloud base, namely $d \sim 10^{3} \mathrm{~m}$ and $w \sim 1 \mathrm{~m} \mathrm{~s}^{-1}$, leading to the buoyancy parameter, $B \sim 0.1$. Thus the buoyancy parameter is extremely small in typical atmospheric situations.

Another parameter relevant for this purpose is the socalled lazy parameter, $\Gamma$ (assumed to be positive), introduced by Morton (1959; see also Scase et al., 2006). The definition is rather involved, so it is not reproduced here, but it is defined in such a manner that we have $\Gamma=1$ when a plume satisfies a similarity solution of Morton et al. (1956). Here, again, it is important to realize that the classical similarity solution assumes a finite vertical velocity (momentum) at a source. Only when the initial ratio between the momentum and the mass satisfies a particular value, $\Gamma=1$, is the similarity solution obtained. Otherwise, the plume evolution does not follow that of the similarity solution. For this reason, the 
regime with $\Gamma=1$ is proposed to be called "pure plume". When $\Gamma>1$ (disturbed or lazy), the mass source dominates. When $\Gamma<1$ (forced), the momentum source dominates. The "lazy" situation is expected to be more relevant to the atmospheric plumes for the reason just discussed.

These short considerations suggest that classical laboratory-plume experiments are not necessarily relevant for atmospheric plumes. Here atmospheric convection is in a qualitatively different regime than for the typical laboratory-experiment convective plumes. The bubble dynamics more relevant for atmospheric convection under small buoyancy parameters have been studied through experiment, for example by Sànchez et al. (1989).

\section{Role of the plume hypothesis in the mass-flux formulation}

As a historical review suggests so far, it may even be argued that the steady-plume model is introduced into the mass-flux convection parameterization more by a historical "accident". We may point out the strong role of Joanne Malkus Simpson in this process. It is then reasonable to ask how strongly the mass-flux formulation is constrained by this steady-plume hypothesis.

The steady-plume hypothesis has two major consequences for the standard mass-flux parameterization formulation. These are

1. separation of the variables;

2. determination of its vertical structure by an entrainment-detrainment hypothesis.

In order to examine these consequences critically, a short summary of the mass-flux formulation is warranted (cf., Yano, 2014). As its name suggests, a key variable in its formulation is the convective mass flux, $M$. Once this variable has been determined, all the output variables for mass-flux convection parameterization can be evaluated in a more or less straightforward manner.

The mass flux, $M$, is in turn determined in two steps by assuming a separation of variables into time and vertical dependences:

$M(z, t)=M_{\mathrm{B}}(t) \eta(z)$.

Here a subscript, $\mathrm{B}$, is added to the time-dependent part, because it is customarily defined as the mass-flux value at the convection base.

We may trace the separation of the variables to the steadyplume hypothesis introduced to the mass-flux convection parameterization under a historical process, as reviewed so far. This hypothesis allows us to assume that the vertical structure, $\eta(z)$, is defined under a steady-plume model, whereas its time-dependent amplitude, $M_{\mathrm{B}}(t)$, is determined by a large-scale environment to which convective processes are slaved, in a similar manner as a gradually modified stratification within a water tank changes the behavior of the plume. The latter is defined under a formal procedure called "closure" (cf., Yano et al., 2013).

However, we should also realize that separation of the variables also comes naturally into the mass-flux formulation when an asymptotic limit of vanishing fractional area for convection is introduced, as a standard limit. It may also be emphasized that the separation of variables is essentially introduced in Arakawa and Schubert (1974) in the latter manner. Discussions on the plume only come later in this paper. Thus, as a logical construction of the mass-flux formulation, the separation of variables does not hang on the plume hypothesis. The idea can be treated in a more abstract manner, probably keeping the term "plume" only as a metaphor.

On the other hand, the vertical structure of the mass flux, $\eta(z)$, is, without exception, determined by invoking the plume dynamics in the current operational schemes. Recall that Morton et al. (1956) proposed an entraining plume, whose vertical structure is determined by

$\frac{1}{\eta} \frac{\mathrm{d} \eta}{\mathrm{d} z}=\frac{1}{M} E$,

with the entrainment rate, $E$, assumed to be proportional to the mass flux, $M$. Here $E / M$ is called the fractional entrainment rate.

The above formulation can be generalized by adding the detrainment, $D$, so that

$\frac{1}{\eta} \frac{\mathrm{d} \eta}{\mathrm{d} z}=\frac{1}{M}(E-D)$.

From the point of view of the plume dynamics, this is the most general manner for determining a vertical structure of convection. There are extensive debates on the procedure for determining the convection profile under this framework, as already discussed in Sect. 5.

However, once we accept the fact that the entrainmentdetrainment formalism is merely a historical "accident", there is no longer a strong reason for upholding it. A vertical structure of mass flux may be determined by any other different manner.

Unfortunately, there are not many alternative options immediately visible, but one clear choice is to adopt a spectrum representation for the mass flux, $M_{i}$, with the subscript $i$ standing for a convection type. The most classical choice for a set of convective types would be to take a spectrum of entraining plumes with varying fractional entrainment rates, as originally proposed by Arakawa and Schubert (1974).

The principle of separation of variables becomes

$M_{i}(z, t)=M_{i, \mathrm{~B}}(t) \eta_{i}(z)$,

with $i=1, \ldots, N$ when $N$ convection types are considered, based on different entrainment rates, for example. Once such a spectrum formulation has been adopted, we can avoid the 
issue of determining a vertical structure for each type by simply introducing a sufficient number of prescribed vertical profiles, $\left\{\eta_{i}\right\}$. For example, a prescribed entrainment rate would determine the vertical profile simply by vertically integrating Eq. (3). An alternative choice could be simply to take a series of half-sinusoidal shapes with varying heights. In the latter case, the top height of a half-sinusoidal function designates the convection type, $i$.

The key issue is then turned into that of determining the mass-flux spectrum, $\left\{M_{i, \mathrm{~B}}\right\}$, by a closure condition. Arakawa and Schubert's original convective quasi-equilibrium hypothesis is exactly designed to address the problem in this manner. Unfortunately, not much formal investigation of this formulation is reported in the literature (cf., Yano and Plant, 2012).

There is a further benefit to taking a spectrum of convection: there is no longer a need to introduce the steady-plume hypothesis to individual plumes. Under this generalization, the individual plumes could be totally transient, freely evolving in the fast convective scale. The only requirement is maintaining quasi steadiness of the total thermodynamic tendency of those plumes collectively, as discussed more extensively in Yano (2014): see especially Eq. 7.5. This liberty for introducing transient behaviors under a spectrum formulation becomes even more important when the bubbles are adopted in place of the plumes, as discussed next.

\section{Future perspectives: rediscovery of bubble?}

After this long historical tour, it appears to me that we are turning to a period of rediscovery of the bubble. With the dramatic progress of digital computational power, it is now possible to simulate details of the atmospheric convective dynamics with relative ease. At such a level of detail, convection is clearly not steady, but rather transient. As a result, it is far easier to recognize atmospheric convection consisting of an ensemble of bubbles, rather than of a quasi steady plume.

Under this trend, extensive process studies focused on bubbles have begun to appear (e.g., Sherwood et al., 2013, George Craig, personal communication 2012; Alison Stirling, personal communication 2013), and more are likely to follow. Improvements in laboratory experiment technologies with sophisticated measurements based on laser technology (cf., Korczyk et al., 2012) are equally remarkable. An image from their experiments (Fig. 5) suggests the extent of the details available: these experiments can measure, with the help of particle image velocimetry (PIV; Korczyk et al., 2006), much finer details of the flows associated with a bubble than current LESs can: see also Diwan et al. (2014).

We could learn a lot along this line of research in the years to come. Here, we may even say that we have come a long way to recognize correctly again that convection consists of bubbles. However, this is somewhat ironic after years

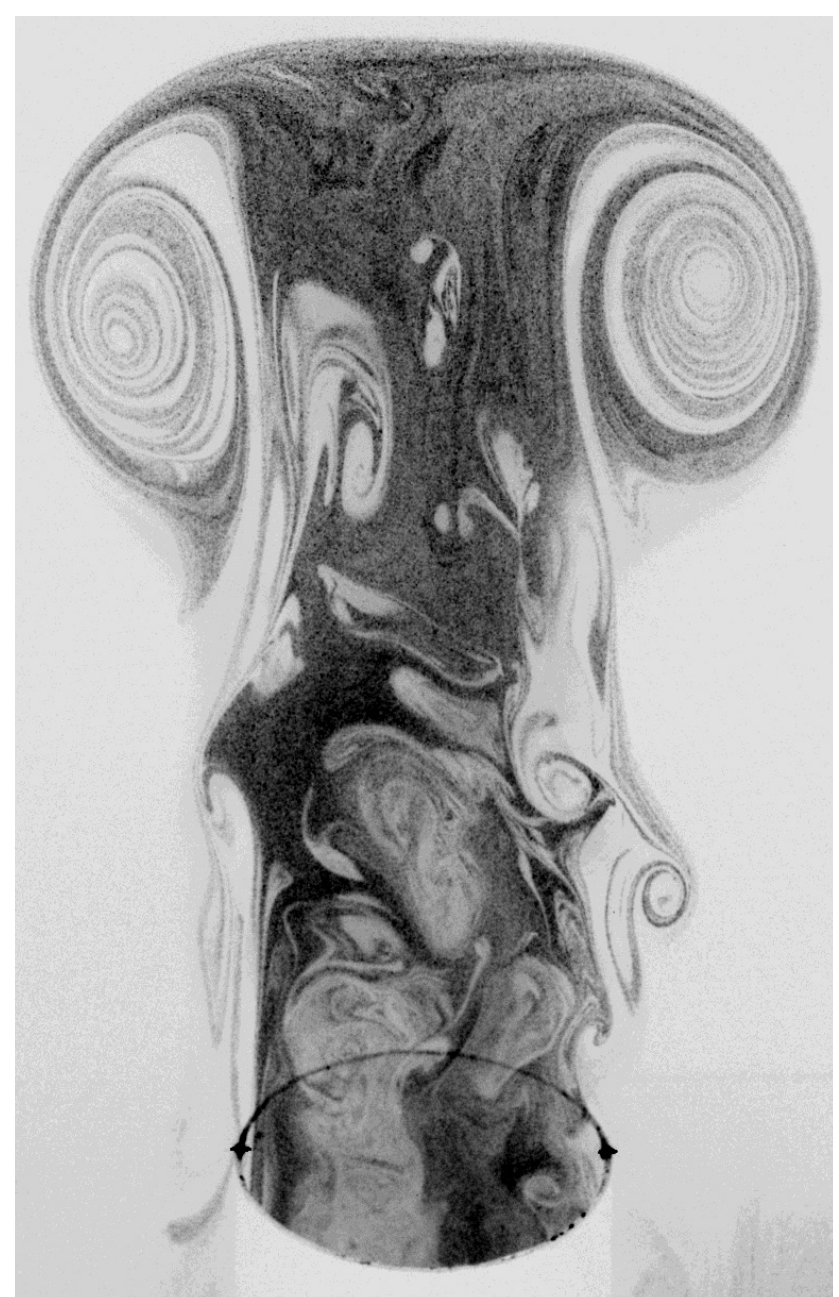

Figure 5. A cross section of a thermal plume generated in a laboratory with use of a humidifier as a buoyancy source. Distribution of condensed water is shown by gray tones (courtesy: Anna Gorska and Szymon Malinowski).

of efforts to develop convection parameterization based on a steady-plume hypothesis.

Keep in mind that generally it is not legitimate simply to criticize a certain parameterization by saying that it neglects a key element in a given process, such as the transient nature of real atmospheric convection consisting of bubbles. The goal of a parametrization is not to reproduce the whole structure of a given process accurately. Its only concern is to provide the feedback of the given process in a grid-box average (i.e., large scale). Though a given element may well be important for reconstructing the whole structure of a given process, say in explicit simulations, it may not be as important for a feedback to the large scale. Said differently, the feedback itself may be well described without taking into account such a feature (cf., Yano et al., 2012).

The importance of the transient nature of convection in parameterization must also be considered in this manner. 
Though everyone would agree that atmospheric convection is highly transient, there is no robust evidence to believe that it is crucial to implement this aspect in a parameterization.

As reviewed in the last section, the current convection parameterization based on a steady-plume hypothesis is developed in a self-consistent manner. Thus, such a criticism is like trying to discredit the quasi geostrophic theory based on the fact that it neglects gravity waves. Most of the dynamists would agree that the value of the quasi geostrophic theory is hardly diminished by the fact that it neglects gravity waves. The same could equally be true of the mass-flux convection parameterization: its neglect of convective transiency does not necessarily automatically discredit its value. In this very respect, it is not quite clear how the bubble-dynamics studies contribute to the improvements in mass-flux convection parameterization.

If we are going to re-adopt the bubbles as the basic elements of convection in a diligent manner, then we need to develop a completely new framework for convection parameterization not based on the steady-plume hypothesis, either as an extension of mass flux formulation or by an alternative approach. Recall that Ooyama (1971) claimed that he took the bubbles as the basic elements, but this was only applied under a steady state. Thus, his formalism is reduced to that of the steady plumes in the end (see also Ooyama, 1972). If the bubble paradigm is to be fully accepted, a fully transient description of convection must be developed for a parameterization purpose.

It is important to note that the plume and the bubble are governed by different sets of equations even in the simplest cases: compare Eq. (2) of Morton et al. (1956) and Eqs. (1), (3), and (4) of Turner (1963b). Note that their systems are described in terms of a vertical coordinate and time, respectively. However, even when the latter is transformed into a vertical coordinate, it does not reduce to the former, simply because different physics are considered. Though one may intuitively argue that a stream of bubbles behaves in an analogous manner to an entraining plume, the former is described by a different set of equations than the latter. In other words, although both may arguably be based on a certain "parcel" approximation, they are not equivalent.

Another way of looking at the issue is Eq. (3): a vertical structure of the mass flux is defined under a given fractional entrainment rate, which may generally be given by $E / M=\alpha / R$ with $R$ the radius of a cross section. Here, however, we obtain from the laboratory experiments qualitatively different values for the fractional entrainment rate for entraining plume and a spherical bubble: $\alpha \simeq 0.2$ and 0.25 , respectively (cf., Turner, 1969, 1986).

Note that the above argument is made under a hypothesis of non-interaction between the bubbles. Little study has been performed on the interactions between the bubbles, as already noted in Sect. 2. Whether an interacting ensemble of bubbles behaves like an entraining plume as a whole is a highly speculative matter.
As also already emphasized in Sect. 2, the evolution of a bubble is highly transient, in contrast to the quasi steady nature of plumes, thus the most straightforward modifications would be to take an ensemble of bubbles described by Eqs. (1), (3), and (4) of Turner (1963b) in place of an ensemble of steady plumes under a spectrum formulation of mass flux as discussed in the last section.

Under this new formulation, individual bubbles would behave in a transient manner by explicitly taking into account the convective timescale evolution. Under this formulation, a key constraint is to add a hypothesis of "collective steadiness" of those transient bubbles, which states that a total thermodynamic tendency of bubbles must be steady on convective scales in order to ensure the slow evolution of the largescale dynamics, as already suggested in the last section. This is an attractive option that is worthwhile pursuing.

It may well be possible that an ensemble of bubbles can be described under an analogy with a steady plume. However, in this case, a merit of adopting the "bubble hypothesis" becomes more subtle and even implicit. Under such a re-interpretation, the entrainment-detrainment is simply reduced to a tuning parameter. The question is whether any fundamental bubble theory or extensive measurements of bubbles from laboratory experiments can provide anything useful for this "tuning" exercise.

In other words, if we are going to take a steady-plume system, as described in Sect. 7, merely as a mathematical metaphor, there will be no point in discussing any more which point of view is more central between bubble and plume. The entrainment-detrainment rate would simply become a tunable parameter, or something to be estimated from, say, LES without asking about any physical mechanism behind it.

In this manner, we face dichotomous choices. We may stay with a traditional mass-flux formulation originating from the steady-plume hypothesis, but treating the plume more as a metaphor. As an alternative extreme opposite choice, we may pursue a completely new theory based on the statistical dynamics of bubble ensembles. A final answer to the latter may turn out to be a mathematical analog to a steady-plume formulation. However, that is exactly what we still have to find out.

However, it would be better to conclude the present review in a cautious manner rather than to maintain a misleading optimism. A good parallel would be a question of reducing a spectrum of convective plumes under mass-flux formulation into a bulk plume. Though a bulk model is often considered to be an ensemble-averaged version of a spectrum model (e.g., Gregory and Rowntree, 1990), such an interpretation turns out to be difficult to maintain literally (Plant, 2010).

A stepwise generalization of a plume-based formulation could be a third path to take. A general system with such plume dynamics can be constructed by introducing a geometrical constraint consistent with the mass-flux formulation, which may be called segmentally constant approximation 
(SCA), into a cloud-resolving model (CRM: Yano et al., 2005, 2010; Yano, 2012, 2014). Especially when only a single plume is placed over a grid box, a fully prognostic bulk mass flux model can be derived without any further approximations or closures (Yano and Baizig, 2012). This formulation can easily be re-adapted for describing an ensemble of bubbles. Note here that the fractional entrainment rate is $E / M$.

An important lesson from the present historical review is to avoid repeating the mistake of uncritically adopting a formulation already developed for a particular purpose into something else. The bubble vertical-velocity formulation by Levine (1959) was uncritically introduced into the steadyplume problem in this manner. We should avoid the same mistake of reapplying the steady-plume formulation back into an unsteady-bubble problem.

Acknowledgements. The context of COST Action ES0905 is acknowledged. The image for Fig. 5 is provided by Szymon Malinowski. Careful reading of the text by Richard Davy is much appreciated.

\section{Edited by: H. Tost}

\section{References}

Arakawa, A. and Schubert, W. H.: Interaction of a cumulus cloud ensemble with the large-scale environment, Part I, J. Atmos. Sci., 31, 674-701, 1974.

Baines, P. G.: Mixing in flows down gentle slopes into stratified environments, J. Fluid Mech., 443, 237-270, 2001.

Baines, P. G.: Two-dimensional plumes in stratified environment, J. Fluid Mech., 471, 315-337, 2002.

Batchelor, G. K.: Heat convection and buoyancy effects in fluids, Q. J. Roy. Meteor. Soc., 80, 339-358, 1954.

Bechtold, P., Bazile, E., Guichard, F., Mascart, P., and Richard, E.: A mass-flux convection scheme for regional and global models, Q. J. Roy. Meteor. Soc., 127, 869-889, 2001.

Bretherton, C. S., McCaa, J. R., and Grenier, H.: A new parameterization for shallow cumulus convection and its application to marine subtropical cloud-topped boundary layers, Part I: Description and 1-D results, Mon. Weather Rev., 132, 864-882, 2004.

Blyth, A. M., Cooper, W. A., and Jensen, J. B.: A study of the source of entrained air in Montana cumuli, J. Atmos. Sci., 45, 39443964, 1988.

Davies, R. M. and Taylor, G. K.: The mechanics of large bubbles rising through extended liquids and through liquids in tubes, $\mathrm{P}$. R. Soc. London, 200, 375-390, 1950.

Derbyshire, S. H., Maidens, A. V., Milton, S. F., Stratton, R. A., and Willett, M. R.: Adaptive detrainment in a convective parameterization, Q. J. Roy. Meteor. Soc., 137, 1856-1871, 2011.

de Rooy, W. C., Bechtold, P., Frohlich, K., Hohenegger, C., Jonker, H., Mironov, D., Pier Siebesma, A., Teixeira, J., and Yano, J.-I.: Entrainment and detrainment in cumulus convection: an overview, Q. J. Roy. Meteor. Soc., 139, 1-19, doi:10.1002/qj.1959, 2013.
Diwan, S. S., Prasanth P., Sreenivas K. R., Deshpande S. M. and Narasimha, R.: Cumulus-type flows in the laboratory and on the computer: Simulating cloud form, evolution and large-scale structure. Bull. Amer. Meteor. Soc., in press, doi:10.1175/BAMS-D-12-00105.1, 2014.

Donner, L. J.: A cumulus parameterization including mass fluxes, vertical momentum dynamics, and mesoscale effects, J. Atmos. Sci., 50, 889-906, 1993.

Emanuel, K. A.: A scheme for representing cumulus convection in large-scale models, J. Atmos. Sci., 48, 2313-2335, 1991.

Fritsch, U.: Turbulence: The Legacy of A. N. Kolmogorov, Cambridge University Press, 296 pp, 1995.

Gregory, D. and Rowntree, P. R.: A mass flux scheme with representation of cloud ensemble characteristics and stability-dependent closure, Mon. Weather Rev., 118, 1483-1506, 1990.

Holton, J. R.: A one-dimensional cumulus model including pressure perturbations. Mon. Wea. Rev., 101, 201-205, 1973.

Houghton, H. and Cramer, H.: A theory of entrainment in convective currents, J. Meteorol., 8, 95-102, 1951.

Kain, J. S. and Fritsch, J. L.: A one-dimensional entraining/detraining plume model and its application in convective parameterization, J. Atmos. Sci., 47, 2784-2802, 1990.

Kasahara, A. and Asai, T.: Effects of an ensemble of convective elements on the large-scale motions of the atmosphere, J. Meteorol. Soc. Jpn., 45, 280-291, 1967.

Korczyk, P., Malinowski, S. P., and Kowalewski, T. A.: Mixing of cloud and clear air in centimeter scales observed in laboratory by means of Particle Image Velocimetry. Atmos. Res., 82, 173-182, doi:10.1016/j.atmosres.2005.09.009, 2006.

Korczyk, P. M., Kowalewski, T. A., and Malinowski, S. P.: Turbulent mixing of clouds with the environment: Small scale two phase evaporating flow investigated in a laboratory by particle image velocimetry, Physica D, 241, 288-296, 2012.

Kuo, H. L. and Raymond, W. H.: A quasi-one-dimensional cumulus model cloud model and parameterization of cumulus heating and mixing effects. Mon. Wea. Rev., 108, 991-1009, 1980.

Lamb, H.: Hydrodynamics, 6th Ed., Cambridge University Press, 738 pp., 1932.

Levine, J.: Spherical vortex theory of bubble-like motion in cumulus clouds, J. Meteorol., 16, 653-662, 1959.

List, E. J.: Turbulent jets and plumes, Annu. Rev. Fluid Mech., 14, 189-212, 1982.

Ludlam, F. H.: The hail problem, Nublia, 1, 13-96, 1958.

Ludlam, F. H. and Scorer, R. S.: Reviews of modern meteorology -10 , Convection in the atmosphere, Q. J. Roy. Meteor. Soc., 79, 317-341, 1953.

Malkus, J. S.: Recent advances in the study of convective clouds and their interaction with the environment, Tellus, 4, 71-87, 1952.

Malkus, J. S.: Some results of a trade-cumulus cloud investigation, J. Meteorol., 11, 220-237, 1954.

Malkus, J. S. and Scorer, R. S.: The erosion of cumulus towers, J. Meteorol., 12, 43-57, 1955.

Morton, B. R.: Buoyant plumes in a moist atmosphere, J. Fluid Mech., 2, 127-144, 1957.

Morton, B. R.: Forced plumes, J. Fluid Mech., 5, 151-163, 1959.

Morton, B. R.: On Telford's model for clear air convection, J. Atmos. Sci., 25, 135-138, 1968.

Morton, B. R.: Discreet dry convective entities, I: Review, in: The Physics and Parameterization of Moist Atmospheric Convection, 
edited by: Smith, R. K., NATO ASI, Kloster Seeon, Kluwer Academic Publishers, Dordrecht, 143-173, 1997a.

Morton, B. R.: Discreet dry convective entities, II: thermals and deflected jets, in: The Physics and Parameterization of Moist Atmospheric Convection, edited by: Smith, R. K., NATO ASI, Kloster Seeon, Kluwer Academic Publishers, Dordrecht, 175210, 1997b.

Morton, B. R., Taylor, G. I., and Turner, J. S.: Turbulent gravitational convection from maintained and instantaneous sources, $\mathrm{P}$. Phys. Soc., 74, 744-754, 1956.

Ooyama, V. K.: A theory on parameterization of cumulus convection, J. Meteorol. Soc. Jpn., 26, 3-40, 1971.

Ooyama, V. K.: On parameterization of cumulus convection, in: Dynamics of the Tropical Atmosphere, Notes from a Colloquium: Summer 1972: National Center for Atmospheric Research, Boulder, Colorado, 11 July 1972, 496-505, 1972.

Ottino, J. M.: The Kinematics of Mixing: Stretching, Chaos, and Transport, Cambridge Texts in Applied Mathematics, Cambridge University Press, 364 pp., 1989.

Paluch, I. R.: The entrainment mechanism of Colorado cumuli, J. Atmos. Sci., 36, 2467-2478, 1979.

Plant, R. S.: A review of the theoretical basis for bulk mass flux convective parameterization, Atmos. Chem. Phys., 10, 3529-3544, doi:10.5194/acp-10-3529-2010, 2010.

Raymond, D. J.: Observational constraints on cumulus parameterizations, The Representation of Cumulus Convection in Numerical Models, Meteor. Mono., No. 46, Amer. Meteor. Soc., 17-28, 1993.

Raymond, D. J. and Blyth, A. M.: A stochastic mixing model for nonprecipitating cumulus clouds, J. Atmos. Sci., 43, 2708-2718, 1986.

Reuter, G. W.: A historical review of cumulus entrainment studies, B. Am. Meteor. Soc., 67, 151-154, 1986.

Sànchez, O., Raymond, D. J., Libersky, L., and Petschek, A. G.: The development of thermals from rest, J. Atmos. Sci., 46, 2280 2292, 1989.

Saunders, P. M.: Penetrative convection in stably stratified fluids, Tellus, 16, 177-194, 1962.

Scase, M. M., Caufield, C. P., Dalziel, S. B., and Hunt, J. C. R.: Time-dependent plumes and jets with decreasing source strengths, J. Fluid Mech., 563, 443-461, 2006.

Scorer, R. S.: Experiments on convection of isolated masses of buoyancy fluid, J. Fluid Mech., 2, 583-594, 1957.

Scorer, R. S. and Ludlam, F. H.: Bubble theory of penetrative convection, Q. J. Roy. Meteor. Soc., 79, 94-103, 1953.

Scorer, R. S. and Ronne, C.: Experiments with convection bubbles, Weather, 11, 151-154, 1956.

Sherwood, S. C., Hernández-Deckers, D., Colin, M., and Robinson, F.: Slippery thermals and the cumulus entrainment paradox, J. Atmos. Sci., 70, 2426-2442, 2013.

Siebesma, A. P., Bretherton, C. S., Brown, A., Chlond, A., Cuxart, J., Duynkerke, P. G., Jiang, H., Khairoutdinov, M., Lewellen, D., Moeng, C.-H., Sanchez, E., Stevens, B., and Stevens, D. E.: A large eddy simulation intercomparison study of shallow cumulus convection, J. Atmos. Sci., 60, 1201-1219, 2003.

Simpson, J.: On cumulus entrainment and one-dimensional models, J. Atmos. Sci., 28, 449-455, 1971.

Simpson, J.: Reply, J. Atmos. Sci., 29, 220-225, 1972.
Simpson, J.: Cumulus clouds: early aircraft observations and entrainment hypotheses, in: Mesoscale Meteorology - Theories, Observations and Models, edited by: Lilly, D. K. and GalChen, T., 355-373, Reidel, Dordrecht, the Netherlands, $1983 \mathrm{a}$.

Simpson, J.: Cumulus clouds: interactions between laboratory experiments and observations as foundations for models, in: Mesoscale Meteorology - Theories, Observations and Models, edited by: Lilly, D. K. and Gal-Chen, T., 399-412, Dordrecht, the Netherlands, 1983b.

Simpson, J. and Wiggert, V.: Models of precipitating cumulus towers, Mon. Weather Rev., 97, 471-489, 1969.

Simpson, J., Simpson, R. H., Andrews, D. A., and Eaton, M. A.: Experimental cumulus dynamics, Rev. Geophys., 3, 387-431, 1965.

Soong, S.-T.: Numerical simulation of warm rain development in an axisymmetric cloud model. J. Atmos. Sci., 31, 1262-1285, 1974

Squires, P.: The spatial variation of liquid water content and droplet concentration in cumuli, Tellus, 10, 372-380, 1958a.

Squires, P.: Penetrative downdraughts in cumuli, Tellus, 10, 381389, 1958b.

Stommel, H.: Entrainment of air into a cumulus cloud, J. Meteorol., 4, 91-94, 1947.

Stommel, H.: Entrainment of air into a cumulus cloud II, J. Meteorol., 8, 127-129, 1951.

Sud, Y. C. and Walker, G. K.: Microphysics of clouds with relaxed Arakawa-Schubert scheme (McRAS), Part I: Design and evaluation with GATE Phase III data, J. Atmos. Sci., 56, 3196-3220, 1999.

Taylor, G. R. and Baker, M. B.: Entrainment and detrainment in cumulus clouds, J. Atmos. Sci., 48, 112-121, 1991.

Telford, J. W.: The convective mechanism in clear air, J. Atmos. Sci., 23, 652-666, 1966.

Telford, J. W.: Reply, J. Atmos. Sci., 25, 138-139, 1968.

Telford, J. W.: Turbulence, entrainment and mixing in cloud dynamics, Pure Appl. Geophys., 113, 1067-1084, 1975.

Townsend, A. A.: Entrainment and the structure of turbulent flow, J. Fluid Mech., 41, 13-46, 1970.

Turner, J. S.: Buoyant vortex rings, P. R. Soc. London, 239, 61-75, 1957.

Turner, J. S.: The "starting plume" in neutral surroundings, J. Atmos. Sci., 13, 356-368, 1962.

Turner, J. S.: Model experiments relating to thermal with increasing buoyancy, Q. J. Roy. Meteor. Soc., 89, 62-74, 1963 a.

Turner, J. S.: The motion of buoyancy elements in turbulent surroundings, J. Fluid Mech., 16, 1-16, $1963 \mathrm{~b}$.

Turner, J. S.: The flow into an expanding spherical vortex, J. Fluid Mech., 18, 195-208, 1964.

Turner, J. S.: Buoyant plumes and thermals, Annu. Rev. Fluid Mech., 1, 29-44, 1969.

Turner, J. S.: Turbulent entrainment: the development of the entrainment assumption, and its application to geophysical flows, J. Fluid Mech., 173, 431-471, 1986.

Warner, J.: On steady-state one-dimensional models of cumulus convection, J. Atmos. Sci., 27, 1035-1040, 1970.

Warner, J.: Comments "On cumulus entrainment and onedimensional models”, J. Atmos. Sci., 29, 218-219, 1972.

Woodward, B.: The motion in and around isolated thermals, Q. J. Roy. Meteor. Soc., 85, 144-151, 1959.

Yau, M. K., 1979: Perturbation pressure and cumulus convection. J. Atmos. Sci., 36, 690-694. 
Yano, J.-I.: Mass-flux subgrid-scale parameterization in analogy with multi-component flows: a formulation towards scale independence, Geosci. Model Dev., 5, 1425-1440, doi:10.5194/gmd5-1425-2012, 2012.

Yano, J.-I.: Formulation structure of the mass-flux convection parameterization. Dyn. Atmos. Ocean, 67, 1-28, doi:10.1016/j.dynatmoce.2014.04.002, 2014.

Yano, J.-I. and Baizig, H.: Single SCA-plume dynamics, Dyn. Atmos. Ocean, 58, 62-94, 2012.

Yano, J.-I. and Plant, R. S.: Convective quasi-equilibrium, Rev. Geophys., 50, RG4004, doi:10.1029/2011RG000378, 2012.

Yano, J.-I., Redelsperger, J.-L., Guichard, F., and Bechtold, P.: Mode decomposition as a methodology for developing convective-scale representations in global models, Q. J. Roy. Meteor. Soc., 131, 2313-2336, 2005.
Yano, J.-I., Benard, P., Couvreux, F., and Lahellec, A.: NAM-SCA: Nonhydrostatic Anelastic Model under Segmentally-Constant Approximation, Mon. Weather Rev., 138, 1957-1974, 2010.

Yano, J.-I., Cheedela, S. K., and Roff, G. L.: A compressed superparameterization: test of NAM-SCA under single-column GCM configurations, Atmos. Chem. Phys. Discuss., 12, 28237-28303, doi:10.5194/acpd-12-28237-2012, 2012.

Yano, J.-I., Bister, M., Fuchs, Ž., Gerard, L., Phillips, V. T. J., Barkidija, S., and Piriou, J.-M.: Phenomenology of convectionparameterization closure, Atmos. Chem. Phys., 13, 4111-4131, doi:10.5194/acp-13-4111-2013, 2013.

Zhang, J., Lohmann, U., and Stier, P.: A microphysical parameterization for convective clouds in the ECHAM5 climate model: single-column model results evaluated at the Oklahoma Atmospheric Radiation Measurement Program site, J. Geophys. Res. 110, D15S07, doi:10.1029/2004JD005128, 2005. 